\title{
Sensitivity of three-body decays to the reactions mechanism and the initial structure by example of ${ }^{6} \mathrm{Be}$
}

\author{
L. V. Grigorenko, ${ }^{1,2,3}$ I. A. Egorova, ${ }^{4}$ R. J. Charity, ${ }^{5}$ and M. V. Zhukov ${ }^{6}$ \\ ${ }^{1}$ Flerov Laboratory of Nuclear Reactions, JINR, Dubna, RU-141980 Russia \\ ${ }^{2}$ GSI Helmholtzzentrum für Schwerionenforschung, Planckstraße 1, D-64291 Darmstadt, Germany \\ ${ }^{3}$ National Research Center “Kurchatov Institute,” Kurchatov Square 1, RU-123182 Moscow, Russia \\ ${ }^{4}$ Bogoliubov Laboratory of Theoretical Physics, JINR, Dubna, 141980 Russia \\ ${ }^{5}$ Department of Chemistry, Washington University, St. Louis, Missouri 63130, USA \\ ${ }^{6}$ Fundamental Physics, Chalmers University of Technology, S-41296 Göteborg, Sweden
}

(Received 23 November 2012; published 28 December 2012)

\begin{abstract}
The influence of the initial-state structure and the reaction mechanism on three-body decays is investigated using the example of the ${ }^{6} \mathrm{Be}$ continuum populated in neutron-knockout reactions on ${ }^{7} \mathrm{Be}$. The sensitivity of the ${ }^{6} \mathrm{Be}$ excitation spectrum and the three-body correlations to the different components of the model is demonstrated. It is shown that the spin composition of the initial state may have an overwhelming effect on the three-body continuum. The characteristics and structure of the second $0_{2}^{+}$and $2_{2}^{+}$states in ${ }^{6} \mathrm{Be}$ are predicted and the conditions for their reliable observations are formulated. The effects of interference and the alignment of three-body states on the three-body correlations are demonstrated.
\end{abstract}

DOI: 10.1103/PhysRevC.86.061602

PACS number(s): 25.10.+s, 23.50.+z, 21.60.Gx, 24.70.+s

Introduction. The study of systems beyond nuclear driplines is an important trend in modern low-energy nuclear research. Many of these systems belong to the three-body or even few-body continuum, and the reaction theory for populating these states is not well developed. Modern high-precision experiments with exotic beams require complicated analyses and advanced theoretical treatment, and call for deeper insights in this field.

Observables in reactions producing unbound systems depend on three major ingredients: (i) the structure of initial nuclei, (ii) the reaction mechanism, and (iii) the final-state interaction (FSI). For very narrow resonances (extremely long-lived states), the aspects (i) and (ii) lose importance as the structure formed in the reaction has enough time to "forget" how it was created. Then for a consistent description of the system, it is sufficient to study only the decay process (FSI effects) by itself. However, exactly when this approach becomes valid is not always clear in advance. Clarity in this issue is especially important for systems beyond the driplines, where the resonant states (often already the ground states) are quite broad.

In this work, we demonstrate the importance of the reaction mechanism and the initial-state structure for investigations of few-body systems beyond the driplines using the example of ${ }^{6} \mathrm{Be}$ (three-body $\alpha+p+p$ continuum) populated in neutronknockout reactions from ${ }^{7} \mathrm{Be}$ projectiles. The first result of these studies has been published in Ref. [1], elucidating the mechanism of democratic decay. Good agreement with experimental data was demonstrated for both the excitation spectrum and the three-body energy-angular correlations over a broad range of excitation energy. However, in this compact experimental work, many important theoretical issues of broader interest were left aside. In the present work, we focus on the most interesting theoretical results arising from our studies of the data [1]. This is a timely message considering the recent interest in the ${ }^{6} \mathrm{Be}$ system [2-6]. We also think that these results should have an important impact on our understanding of three-body decays in general and aid in formulating experimental strategies for studies of this phenomenon.

Theoretical model. The major features of the theoretical model were described in Ref. [1], but some details are needed in the context of this work. The three-body $\alpha+p+p$ final-state interactions forming the continuum of ${ }^{6} \mathrm{Be}$ are described by solving the inhomogeneous three-body Schrödinger equation,

$$
\left(\hat{H}_{3}-E_{T}\right) \Psi^{(+)}=\Phi_{\mathbf{q}},
$$

where $\Psi^{(+)}$is a wave function (WF) with pure outgoing asymptotics obtained with the approximate boundary conditions of the three-body Coulomb problem [2,3]. The energy $E_{T}$ is relative to the $\alpha+p+p$ threshold. The source term $\Phi_{\mathbf{q}}$ depends on the vector $\mathbf{q}$ of the transferred momentum and contains information about the precursors and the reaction mechanism. The knockout of a neutron from ${ }^{7} \mathrm{Be}$ is described as a sudden neutron removal. After acting with the neutron annihilation operator on the ${ }^{7} \mathrm{Be} \mathrm{WF}$ in coordinate space, we obtain

$$
\Phi_{\mathbf{q}}=\int d^{3} r_{n} e^{i \mathbf{q r}_{n}}\left\langle\Psi_{{ }^{4} \mathrm{He}} \mid \Psi_{7_{\mathrm{Be}}}\right\rangle .
$$

Here vector $\mathbf{r}_{n}$ points to the removed neutron; see Fig. 1.

The ${ }^{7} \mathrm{Be} \mathrm{WF}$ is constructed as an "inert" $\alpha$-core plus $p$-wave neutron and two protons with coupling $\left[l_{j}(v)\left[l_{j}\left(\pi_{1}\right) l_{j}\left(\pi_{2}\right)\right]_{J}\right]_{J_{7 \mathrm{Be}}}$. The overlap integral with the $\alpha$ particle for this WF is

$$
\begin{aligned}
\left\langle\Psi_{{ }^{H} \mathrm{He}} \mid \Psi_{7_{\mathrm{Be}}}\right\rangle= & \alpha\left[p_{3 / 2}\left[p_{3 / 2}^{2}\right]_{0}\right]_{3 / 2}+\beta\left[p_{3 / 2}\left[p_{1 / 2}^{2}\right]_{0}\right]_{3 / 2} \\
& +\gamma\left[p_{3 / 2}\left[p_{3 / 2}^{2}\right]_{2}\right]_{3 / 2} \\
& +\delta\left[p_{3 / 2}\left[\frac{p_{3 / 2} p_{1 / 2}-p_{1 / 2} p_{3 / 2}}{\sqrt{2}}\right]_{2}\right]_{3 / 2} .
\end{aligned}
$$

After neutron removal, the terms with coefficients $\{\alpha, \beta\}$ lead to the population of the $0^{+}$state in ${ }^{6} \mathrm{Be}$ while the terms with 


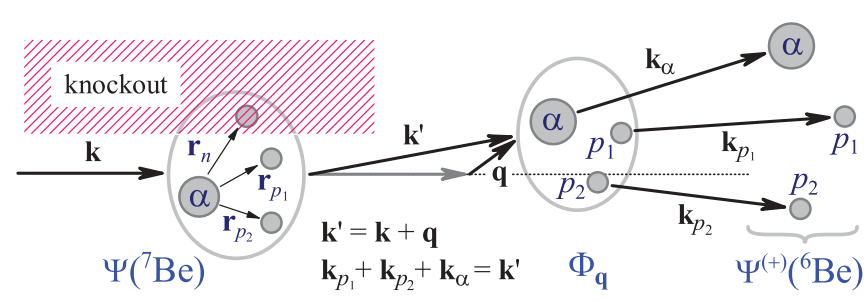

FIG. 1. (Color online) Schematic illustrating the ${ }^{6} \mathrm{Be}$ population formed by knocking a neutron out of a ${ }^{7} \mathrm{Be}$ projectile.

coefficients $\{\gamma, \delta\}$ lead to the population of the $2^{+}$state. Within the $0^{+}$and $2^{+}$configurations, the ratios $\alpha / \beta$ and $\gamma / \delta$ define the spin composition of $\Phi_{\mathbf{q}}$, namely, the probability $W_{S}$ of configurations with definite total spin $S$ (coupled spins of the two "valence" protons). The components of the source function with definite total angular momentum $J$ can be written as

$$
\Phi_{J, q} \sim \sqrt{1-\eta_{J}^{2}}|J, S=0\rangle+\eta_{J}|J, S=1\rangle,
$$

where the coefficients $\eta_{J}$, controlling the $W_{S=0} / W_{S=1}$ ratio, can be expressed via $\{\alpha, \beta, \gamma, \delta\}$ using coefficients from Table I.

For single-particle motion, we use the harmonic-oscillator WFs whose radial behavior is

$$
\phi(r)=(8 / 3 \sqrt{\pi})^{1 / 2}\left(r^{2} / r_{0}^{5 / 2}\right) \exp \left[-r^{2} /\left(2 r_{0}^{2}\right)\right] .
$$

The value $r_{0}=1.8 \mathrm{fm}$ was used in the calculations, which corresponds within our model to the experimental matter radius of 2.31(2) fm for ${ }^{7} \mathrm{Be}$.

The differential cross section is expressed via the flux induced by the WF $\Psi^{(+)}$on the remote surface $S$

$$
\left.\frac{d \sigma}{d^{3} k_{\alpha} d^{3} k_{p_{1}} d^{3} k_{p_{2}}} \sim\left\langle\Psi^{(+)}|\hat{j}| \Psi^{(+)}\right\rangle\right|_{S} .
$$

For the reaction considered, this can be rewritten in terms of the density-matrix formalism

$$
\begin{aligned}
\frac{d \sigma}{d q d E_{T} d \Omega_{5}}= & \sum_{J M, J^{\prime} M^{\prime}} \rho_{J M}^{J^{\prime} M^{\prime}}\left(q, E_{T}\right) \\
& \times A_{J^{\prime} M^{\prime}}^{\dagger}\left(E_{T}, \Omega_{5}\right) A_{J M}\left(E_{T}, \Omega_{5}\right) .
\end{aligned}
$$

Some ingredients of Eqs. (6) and (7) are illustrated in Fig. 1. In Eq. (7), the contributions of the three-body dynamics (amplitudes $A_{J M}$ ) and the reaction mechanism (density matrix $\left.\rho_{J M}^{J^{\prime} M^{\prime}}\right)$ are explicitly separated. For direct reactions, the density matrix has an especially simple form in the frame with the $z$ axis coinciding with the direction of the transferred momentum q. We use two limiting forms of the density matrix:

$$
\rho_{00}^{00}=1, \quad \rho_{2 M}^{2 M}=1 / 5, \quad \rho_{20}^{00}=\rho_{00}^{20}=\cos \left(\phi_{20}\right) / \sqrt{5},
$$

TABLE I. The recoupling coefficients from shell-model-like $(j j)$ coupling in the source to $(l s)$ coupling of the three-body model.

\begin{tabular}{lcccc}
\hline \hline$j_{1}, j_{2}, J$ & $3 / 2,3 / 2,0$ & $1 / 2,1 / 2,0$ & $3 / 2,3 / 2,2$ & $3 / 2,1 / 2,2$ \\
\hline$S=0$ & $\sqrt{2 / 3}$ & $\sqrt{1 / 3}$ & $\sqrt{1 / 3}$ & $\sqrt{2 / 3}$ \\
$S=1$ & $-\sqrt{1 / 3}$ & $\sqrt{2 / 3}$ & $\sqrt{2 / 3}$ & $-\sqrt{1 / 3}$ \\
\hline \hline
\end{tabular}
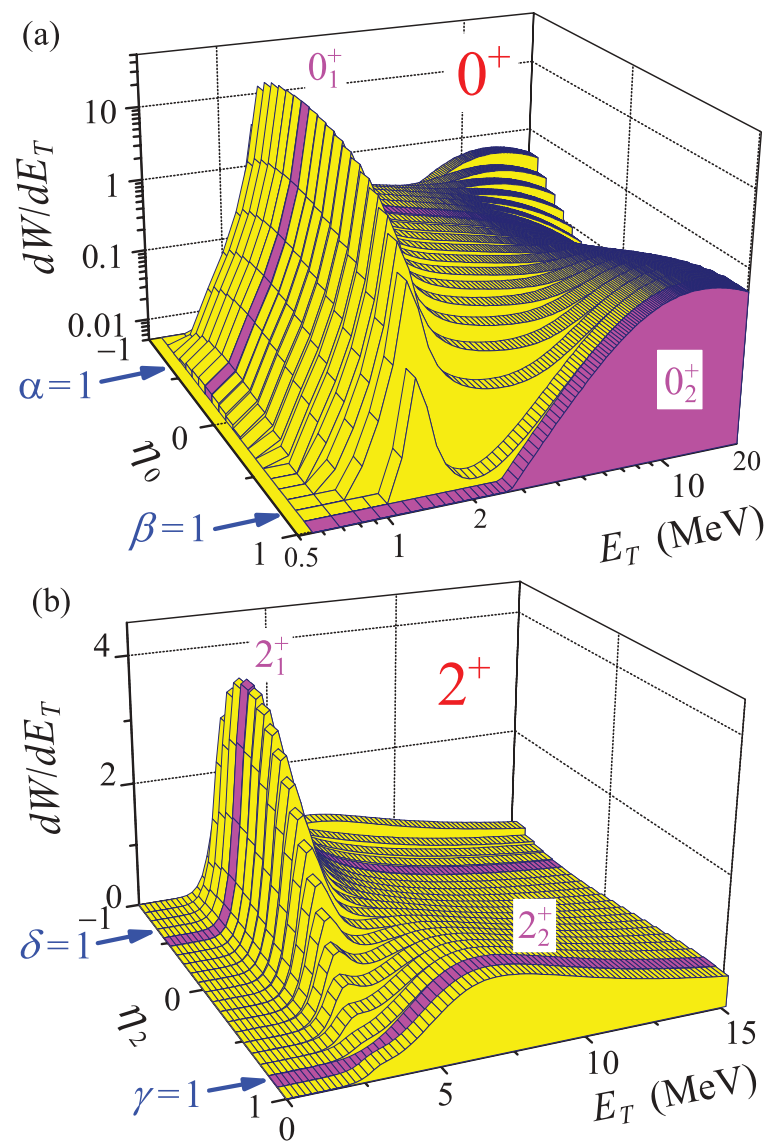

FIG. 2. (Color online) Excitation spectra of (a) $0^{+}$and (b) $2^{+}$ states as function of the spin composition of the source function. The magenta (gray)-colored marked grids correspond to the first and second excitations $J_{1}^{+}$and $J_{2}^{+}$; see Fig. 4. Arrows show the positions corresponding to definite shell-model configurations in the source.

$$
\rho_{00}^{00}=1, \quad \rho_{20}^{20}=1, \quad \rho_{20}^{00}=\rho_{00}^{20}=\cos \left(\phi_{20}\right) .
$$

In the sudden-removal model of Eq. (2), there is no alignment of the final state. This should lead to Eq. (8) with the relative phase of the $0^{+}$and $2^{+}$states $\phi_{20}=\pi$. However, it is clear that some alignment should be introduced by a realistic reaction mechanism. To check our sensitivity to this, we also used the density matrix of Eq. (9) corresponding to the completely aligned case and kept $\phi_{20}$ as a parameter in both cases. In order to compare with experimental results, Eq. (7) provides theoretical input for Monte Carlo (MC) simulations used to deal with the bias introduced by experimental apparatus.

The reaction model and the initial-state WF can be seen as simplistic. However, this fits the aim of this paper to provide a "proof-of-concept" demonstration, where interdependencies on different aspects of the model are as transparent as possible.

Sensitivity of the ${ }^{6} \mathrm{Be}$ spectrum to $W_{S=0} / W_{S=1}$ is demonstrated in Fig. 2. For some range of parameters, the conventional picture of ${ }^{6} \mathrm{Be}$ excitation is observed with the $0^{+}$and $2^{+}$states at 1.37 and $3.05 \mathrm{MeV}$, respectively, with significant variations taking place only in the high-energy "tail" of the distribution. However, with other variations of the parameters, the "normal" states of ${ }^{6} \mathrm{Be}$ "fade" and even completely 


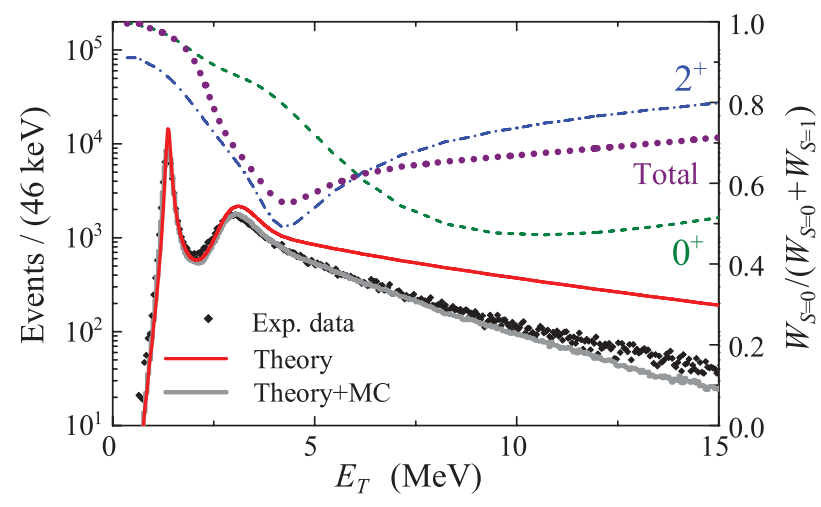

FIG. 3. (Color online) Left axis: theoretical ${ }^{6} \mathrm{Be}$ excitation spectrum together with the results of the MC simulations superimposed on the data [1]. Right axis: predicted evolution of the spin composition in ${ }^{6} \mathrm{Be}$ for $0^{+}, 2^{+}$, and the total spectrum fitted to experimental data (the sum of $0^{+}$and $2^{+}$contributions).

disappear, while new broad peaks arise for these two states at $\sim 12$ and $\sim 7 \mathrm{MeV}$, respectively. Thus, our reaction model links variations of the ${ }^{6} \mathrm{Be}$ excitation spectrum to variations of the structure of ${ }^{7} \mathrm{Be}$. In this work we investigate this link quantitatively without reference to realistic structure of ${ }^{7} \mathrm{Be}$ in order to find boundaries for the possible scale of effects and establish principal opportunity to use three-body-continuum spectra as tools to study the spectroscopy of the precursor.

The broad range of spectra obtained for the $0^{+}$and $2^{+}$ continuum provide an opportunity to fit the experimental spectrum of ${ }^{6} \mathrm{Be}$. This fit, obtained in Ref. [1], is shown in Fig. 3 together with the MC and experimental data. It corresponds to the simple case of pure $S=0$ population in the source function $\left(\eta_{J}=0\right)$ corresponding to a parameter set for initial state of $\{\alpha, \beta, \gamma, \delta\}=\{0.42,0.3,0.49,0.7\}$. It should be noted that the spin content of the final state may have nothing in common with the spin content of the source function as the spin quantum number is not conserved by the Hamiltonian of Eq. (1). The predicted final-state spin composition (Fig. 3, right axis) evolves rapidly between the location of the $0^{+}$peak and past the location of the $2^{+}$peak, indicating important modifications of the nuclear structure in this energy region.

Second $0^{+}$and $2^{+}$states. The above observations allow us to determine the properties of the $0_{2}^{+}$and $2_{2}^{+}$states. We just need to choose the parameter settings minimizing population of the normal resonant peaks. An important feature of "pure" first and second states, illustrated in Fig. 4 (see also arrows in Fig. 2), is that the spin composition depends weakly on energy. The existence of a common spin structure independent of energy allows us to interpret $J_{1}^{+}$and $J_{2}^{+}$as different states, although they can be represented by (relatively) broad overlapping structures. This property of "pure" states is in sharp contrast with the spin evolution for the "composite" situation of Fig. 3. The spin-content ratios $0_{1}^{+} / 0_{2}^{+}$and $2_{1}^{+} / 2_{2}^{+}$ provide a simple structural idea: These states are partners, in the sense that they are the orthogonal combinations of the $S=0$ and $S=1$ configurations. For example, for $2^{+}$states it can be seen that the $W_{S=0} / W_{S=1}$ ratio is $2: 1$ for the first and $\sim 1: 2$ for the second resonance. It can be seen from Figs. 2 and 4 that the "pure" states are best populated from source functions
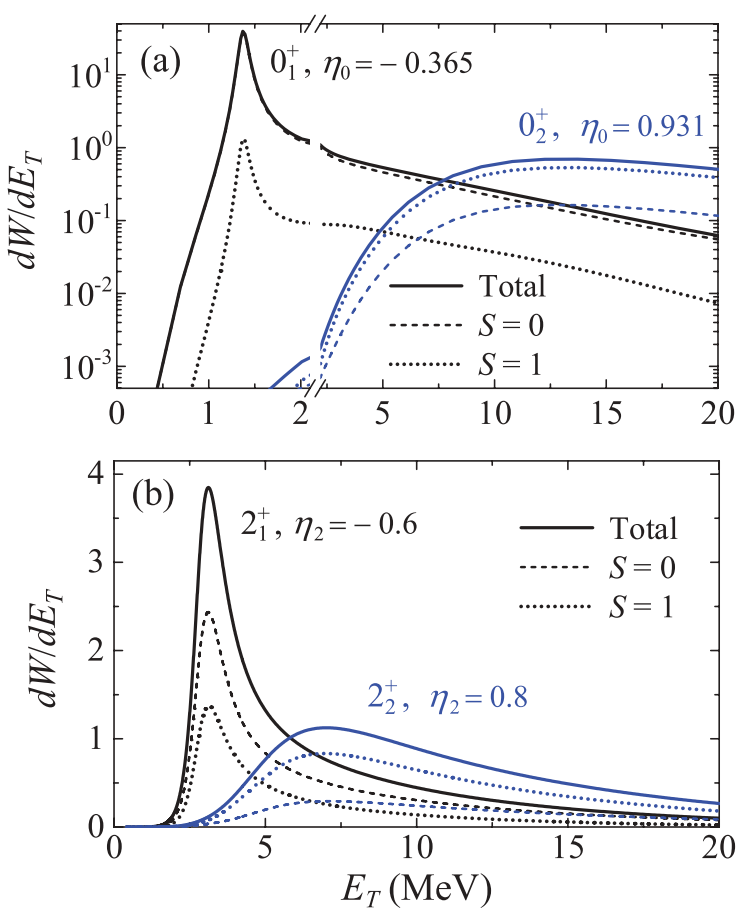

FIG. 4. (Color online) Excitation spectra and spin composition for the ${ }^{6} \mathrm{Be}$ continuum explicitly representing the first and second resonances for (a) $J^{\pi}=0^{+}$and (b) $2^{+}$. Note the broken abscissa in panel (a).

which are close to pure shell configurations in Eq. (3) and in fact their structure is reasonably close to such pure shell configurations.

The above insight on the structure of the $0_{2}^{+}$and $2_{2}^{+}$states of ${ }^{6} \mathrm{Be}$ provides clear guidelines for experimental searches: The reaction mechanism should enrich the $S=1$ component in the final state.

Radial dependence. The sensitivity to the radial characteristics of the source is presented in the Fig. 5. It is practically nonexistent for $0_{1}^{+}$: Variations take place only in the "tail," a few decay widths higher than the resonance position. The $2_{1}^{+}$ sensitivity is quite small: The resonance width is affected on the level of $15 \%$. However, with increasing excitation energy of the states (and hence with increasing decay width) the effect grows. There is about $0.8-\mathrm{MeV}$ uncertainty of the $2_{2}^{+}$position connected with the radial extent of the source, and the profile of the cross section is strongly affected. Variation of $0_{2}^{+}$properties is so large (few million electron volts in peak position) that the properties of such a state cannot be discussed without a detailed account of the reaction mechanism.

Three-body correlations. The three final momentum vectors in Eq. (6) and Fig. 1 can be transformed to the transferred momentum $\mathbf{q}$ and the Jacobi momenta $\mathbf{k}_{x}, \mathbf{k}_{y}$ in Eq. (7):

$$
\begin{aligned}
& \mathbf{k}_{x}=\frac{A_{2} \mathbf{k}_{1}-A_{1} \mathbf{k}_{2}}{A_{1}+A_{2}}, \quad \mathbf{k}_{y}=\frac{A_{3}\left(\mathbf{k}_{1}+\mathbf{k}_{2}\right)-\left(A_{1}+A_{2}\right) \mathbf{k}_{3}}{A_{1}+A_{2}+A_{3}}, \\
& E_{T}=E_{x}+E_{y}=k_{x}^{2} / 2 M_{x}+k_{y}^{2} / 2 M_{y},
\end{aligned}
$$

where $M_{x}$ and $M_{y}$ are the reduced masses of the $X$ and $Y$ subsystems; see Ref. [3] for details. 

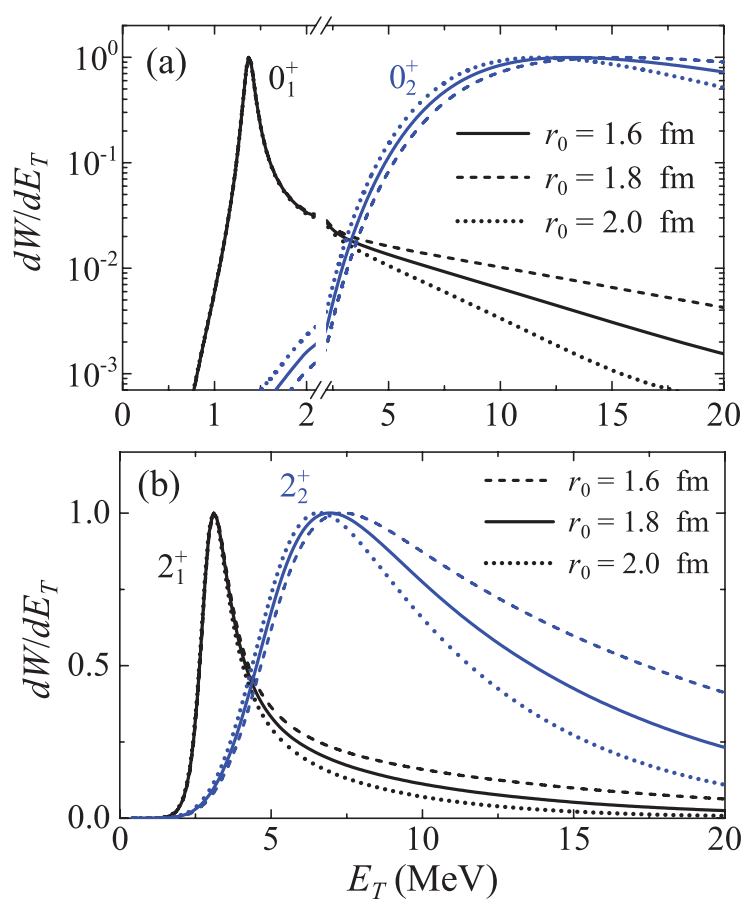

FIG. 5. (Color online) Dependence of the ${ }^{6} \mathrm{Be}$ excitation spectra on the radius parameter $r_{0}$ of the ${ }^{7} \mathrm{Be} \mathrm{WF}$. All spectra are normalized to a maximum value of unity.

The orientation of $\mathbf{q}$ is not a dynamical variable of the model Eq. (2) and thus is not present in Eq. (7). The five-dimensional "hyperspherical solid angle" $\Omega_{5}$ includes two degrees of freedom describing the "internal correlations" of the three-body system, which are ordinarily considered as being completely defined by the dynamics of the three-body motion itself. The parameters

$$
\varepsilon=E_{x} / E_{T}, \quad \cos \left(\theta_{k}\right)=\left(\mathbf{k}_{x} \cdot \mathbf{k}_{y}\right) /\left(k_{x} k_{y}\right)
$$

provide what we call "complete energy-angular correlations." For $k_{3} \rightarrow k_{\alpha}$ we get the "T" Jacobi system, where $\varepsilon$ describes the energy correlation in the $p$ - $p$ channel. For $k_{3} \rightarrow k_{p_{i}}$, the correlations are obtained in one of two possible $(i=1,2)$ "Y" Jacobi systems, where $\varepsilon$ describes the energy correlation in the $\alpha-p$ channel.

The other three degrees of freedom (Euler angles) define "external correlations" as they describe the orientation of the three-body system as a whole. Correlations for the "external" degrees of freedom are evidently defined by the reaction mechanism.

Alignment and interference. The internal three-body correlations for excited states cannot be separated from external information in any practical experiment. The excited states typically have nonzero $J$, making alignment possible, and they reside on the "tails" of the lower energy excitations so that their amplitudes can interfere. From a theoretical point of view, the inclusive energy $\varepsilon$ distributions should be free of interference effects and experience tell us that the angular $\cos \left(\theta_{k}\right)$ distributions are only weakly affected. However, in experiments, the bias of the apparatus introduces cutoffs and distortions, which may induce correlations via

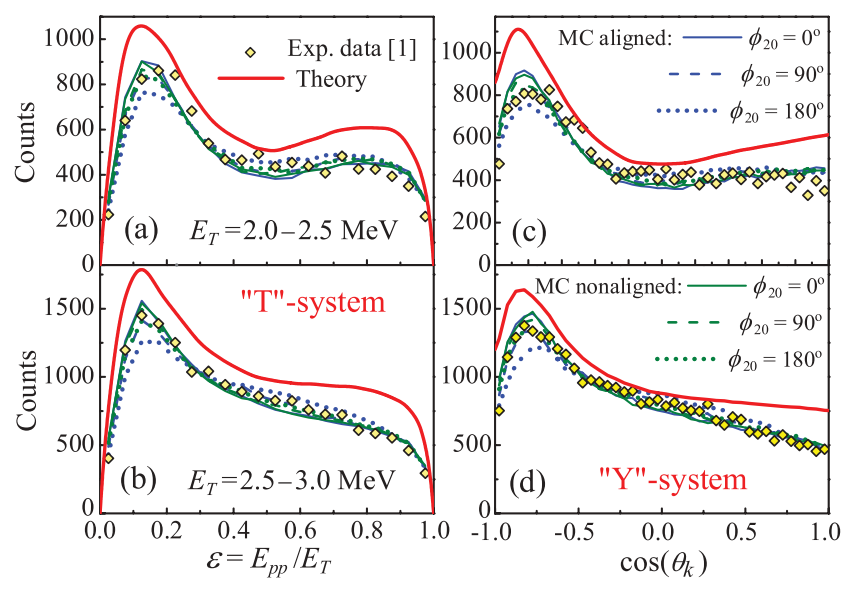

FIG. 6. (Color online) Panels (a) and (b): "Internal" energy distributions in the Jacobi " $T$ " system. Panels (c) and (d): Angular distributions in the Jacobi "Y" system. Upper and lower rows of panels correspond to different $E_{T}$ ranges. The experimental data are shown by the hollow diamonds. Theoretical curves (thick, solid) are given in each panel with some offset to simplify a perception. MC curves for different alignment and interference settings are explained in the legend.

loss of orthogonality for configurations with different angular momenta. Such "induced correlations" are specific for the experiment and should be evaluated by careful MC studies.

A demonstration of alignment and interference effects on experimental results is provided in Fig. 6. As it is clear that the effects are largest for strong $0^{+} / 2^{+}$mixing, we have chosen the $E_{T}=2-3 \mathrm{MeV}$ energy range of the data [1]. MC results for the two limiting cases of completely aligned (9) and "isotropic" (8) density matrices, each with three different phase settings $\phi_{20}=\{0, \pi / 2, \pi\}$, are shown. In Ref. [1], we used the settings of Eq. (8) with $\phi_{20}=\pi$, which is consistent with the data and corresponds to the model of Eq. (2). However, at the moment we cannot exclude that reality is different: Further analysis of the data is needed. Figure 6 shows the energy distribution in the Jacobi " $T$ " system and the angular distribution in the Jacobi "Y" system, which appear to be the most sensitive of the correlation observables. The scale of local variations in the MC distributions is about $20 \%$. The curves are well separated and some deviate considerably from the experimental results.

It should be understood that all the MC curves correspond to the same theoretical distribution and their variations are due to the bias introduced by experimental setup. Thus we conclude that in analyzing high-precision correlation data for excited states where interference and alignment effects become possible, a consistent treatment of the reaction mechanism becomes inevitable. The effect depends strongly on the quality of the experimental setup; it should vanish for an "ideal" instrument. The moderate level of variations in Fig. 6 is connected with the very high efficiency of the setup in Ref. [1]. On lower quality setups, alignment and interference effects can produce very large and poorly controlled modifications. This could be a part of the explanation for the strong deviation of the data of Ref. [5] from the other recent experimental studies $[1,3,6]$. 

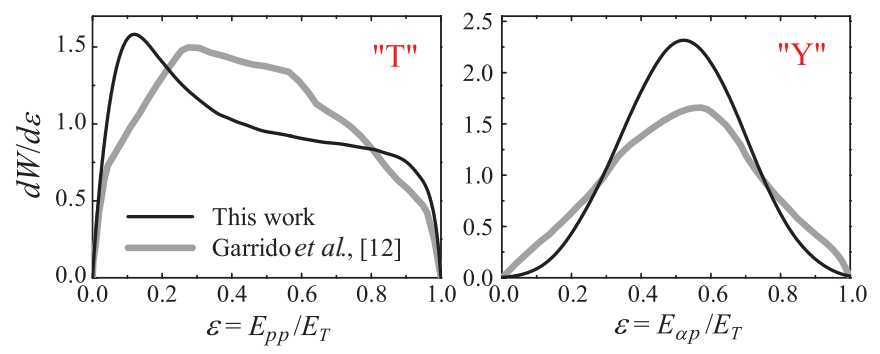

FIG. 7. (Color online) Comparison between the energy distributions for the $2^{+}$resonance of ${ }^{6} \mathrm{Be}$ in " $\mathrm{T}$ " and " $\mathrm{Y}$ " Jacobi systems obtained in the present work and from Ref. [12].

On existence of higher energy negative-parity excitations. The availability of higher excitations in the spectrum of ${ }^{6} \mathrm{Be}[1]$ may drastically affect the proposed interpretation of the data. In Ref. [6], a strong population of presumably $\left\{0^{-}, 1^{-}, 2^{-}\right\}$ states in the charge-exchange reaction induced by $\Delta L=1$ transitions was observed above the first $2^{+}$state. We were cautious about the population of such negative-parity states in the data of Ref. [1]. A dedicated search was performed for asymmetries in certain distributions due to the interference of positive and negative states. However, no significant indication of these asymmetries was found. Also, the dominating shell structure of the ${ }^{7} \mathrm{Be}$ precursor does not imply a strong population of negative-parity states in ${ }^{6} \mathrm{Be}$ within the suddenremoval model. Thus we have confined our interpretation of the ${ }^{6} \mathrm{Be}$ continuum to just the $0^{+}$and $2^{+}$states.

Previous theoretical results on ${ }^{6} \mathrm{Be}$. Three-cluster calculations (microscopic and three-body) for ${ }^{6} \mathrm{Be}$ have been performed in a number of studies [7-12]. In Ref. [9], the energies and widths $\left\{E_{R}, \Gamma\right\}$ of the $0_{2}^{+}$and $2_{2}^{+}$resonances were predicted to be $\{3.5,6.1\}$ and $\{5.2,5.6\} \mathrm{MeV}$. These values are quite different from our predictions $\{\sim 12, \sim 14\}$ and $\{\sim 7, \sim 7\} \mathrm{MeV}$. The reason for this discrepancy is easily understood: Our prediction is that the second $0_{2}^{+}$and $2_{2}^{+}$states are "spin complements" of the first $0_{1}^{+}$and $2_{1}^{+}$states. States of such a nature could not have been obtained in the calculations of Ref. [9] as the $S=1$ component was omitted in their model space.

Among these other theoretical studies, only in Ref. [12] are theoretical correlations (energy distributions for $2^{+}$) presented. The predicted correlations for the $0^{+}$and $2^{+}$states (presumably related to Ref. [12]) are provided in Ref. [5], but in a form which makes them difficult to interpret as only MC results specific for the experimental setup of that work are shown. The distributions of Ref. [12] are not in agreement with our results (see Fig. 7) and hence with the recent highly accurate data of Refs. $[1,6]$. This observation sheds doubts on the applicability of the methods of Ref. [12] to Coulombic three-body decays in general. Our work imposes a new standard of a sophistication required from theoretical calculations to analyze modern highprecision data including correlations.

Conclusions. This work provides important qualitative insights into the question of which aspects of three-body decays can be understood based on the dynamical description of the final state alone and which also require an adequate treatment of the initial state and the reaction mechanism. The major observations of this work are the following:

(i) The population of the ${ }^{6} \mathrm{Be} 0_{1}^{+}$ground state is very stable to variations of the initial-state structure and the reaction mechanism. However, the latter effects become increasingly important with increasing excitation. No sensible description of the continuum above $5-7 \mathrm{MeV}$ can be given without their proper treatment.

(ii) The excitation spectrum of the three-body continuum of ${ }^{6} \mathrm{Be}$ up to $15-20 \mathrm{MeV}$ is found to be very sensitive to the spin composition of the source function in Eq. (1). In our calculations, this was parametrized in terms of the spin content of the precursor. Thus we find that three-body decay can be used as a sensitive instrument of nuclear spectroscopy if the reaction mechanism is well established.

(iii) A procedure to identify the second $0_{2}^{+}$and $2_{2}^{+}$states of ${ }^{6} \mathrm{Be}$ is proposed. These states are found to be "spin complements" of the well-known $0_{1}^{+}$and $2_{1}^{+}$ states, providing a guideline for their experimental observation.

(iv) Alignment and interference effects are observable in experimental data due to the unavoidable experimental bias. They were found to have an important impact on the measured three-body correlations. Proton-proton energy distributions are especially affected. This indicates that caution is needed in studies of $N-N$ correlations in decays of excited (and/or broad) threebody states in general.

Acknowledgments. L.V.G. and I.A.E. are supported by the Helmholtz Association under Grant Agreement IK-RU-002 via FAIR-Russia Research Center. L.V.G. is supported by Russian Foundation for Basic Research 11-02-00657-a and Ministry of Education and Science NS-215.2012.2 grants. R.J.C. is supported by the US Department of Energy, Division of Nuclear Physics, under Grant No. DE-FG02-87ER-40316.
[1] I. A. Egorova et al., Phys. Rev. Lett. 109, 202502 (2012).

[2] L. V. Grigorenko et al., Phys. Lett. B 677, 30 (2009).

[3] L. V. Grigorenko et al., Phys. Rev. C 80, 034602 (2009).

[4] R. J. Charity et al., Phys. Rev. C 82, 041304 (2010).

[5] P. Papka et al., Phys. Rev. C 81, 054308 (2010).

[6] A. S. Fomichev et al., Phys. Lett. B 708, 6 (2012).

[7] B. V. Danilin and M. V. Zhukov, Physics of Atomic Nuclei 56, 460 (1993).
[8] A. Csoto, Phys. Rev. C 49, 3035 (1994).

[9] V. Vasilevsky, A. V. Nesterov, F. Arickx, and J. Broeckhove, Phys. Rev. C 63, 034607 (2001).

[10] P. Descouvemont et al., Nucl. Phys. A 765, 370 (2006).

[11] N. Michel, W. Nazarewicz, and M. Ploszajczak, Phys. Rev. C 82, 044315 (2010).

[12] E. Garrido et al., Nucl. Phys. A 781, 387 (2007). 